\title{
Avaliação do "Acesso de Primeiro Contato" para crianças por meio do PCATool- Brasil
}

\author{
Evaluation of "First Contact Access" for children using the PCATool- Brazil \\ Evaluación del "acceso al primer contacto" de los niños mediante la herramienta PCATool- Brasil
}

Recebido: 08/02/2021 | Revisado: 16/02/2021 | Aceito: 21/02/2021 | Publicado: 28/02/2021

\author{
Rodrigo Balk \\ ORCID: https://orcid.org/0000-0001-5254-6732 \\ Universidade Federal do Pampa, Brasil \\ E-mail: rodrigobalk@unipampa.edu.br \\ Michele Bulhosa de Souza \\ ORCID: https://orcid.org/0000-0003-1818-9537 \\ Universidade Federal do Pampa, Brasil \\ E-mail: michelebulhosa@@unipampa.edu.br \\ Maria Eduarda Perroni Nery \\ ORCID: https://orcid.org/0000-0003-1382-4706 \\ Universidade Federal do Pampa, Brasil \\ E-mail: idudanery@gmail.com
}

\begin{abstract}
Resumo
O estudo teve como objetivo avaliar a qualidade do acesso de primeiro contato na consulta de puericultura em uma Estratégia Saúde da Família (ESF) a partir da perspectiva de cuidadores de crianças, utilizando PCATool como instrumento. Estudo descritivo e transversal, de abordagem quantitativa desenvolvido em ESF de um município da Fronteira-Oeste do Rio Grande do Sul, Brasil. Participaram 34 cuidadores, responsáveis de crianças com idade entre 0 e 5 anos, as quais realizam puericultura na ESF e frequentam a unidade regularmente. A maioria eram mães ou avós (97\%), casadas $(70,6 \%)$, com um filho $(32,4 \%)$ e com casa própria $(82,4 \%)$. Políticas estimulam os serviços para que promovam as consultas de puericultura atingindo todas as crianças de sua população. A fragilidade no acesso da população à consulta de puericultura pode ser percebida em outros estudos que trazem a dificuldade de locomoção e na marcação de dia e horário para consulta como principais motivos da baixa adesão. $\mathrm{O}$ acesso dos indivíduos referente ao atendimento, tempo para conseguir consulta e dificuldade para conseguir atendimento médico obtiveram, em sua grande maioria, respostas satisfatórias. O tempo de espera e aconselhamento pelo telefone obtiveram resultados menores do que os esperados, levantando a hipótese de serem motivos dos quais as famílias possam optar por não dar continuidade ao acompanhamento. Verificou-se necessidade de aprimoramento da equipe na acessibilidade dos usuários à consulta de puericultura, partindo de novos estudos referentes às principais barreiras relacionadas e ações a serem instituídas para ampliar o acesso da população no acompanhamento da criança.
\end{abstract}

Palavras-chave: Atenção primária à saúde; Cuidado da criança; Avaliação em saúde.

\begin{abstract}
The study aimed to evaluate the quality of first contact access in the childcare consultation in a Family Health Strategy (FHS) from the perspective of children's caregivers, using PCATool as an instrument. Descriptive and crosssectional study, with a quantitative approach developed in the FHS of a city in the Fronteira-Oeste of Rio Grande do Sul, Brazil. 34 caregivers participated of the study, which are responsible for children aged 0 to 5 years and whom perform childcare at the FHS and attend the unit. Most were mothers or grandmothers (97\%), married (70.6\%), with one child (32.4\%) and with their own house (82.4\%). Policies encourage services to promote childcare consultations reaching all children in their population. The fragility in the population's access to childcare consultation can be seen in other studies that bring mobility difficulties and in scheduling the day and time for consultation as the main reasons for low adherence. The access of individuals regarding care, time to obtain consultation and difficulty in obtaining medical care obtained, in the great majority, satisfactory answers. The waiting time and telephone counseling obtained less than expected results, raising the hypothesis that they are reasons for the families may choose not to continue the monitoring. There is a need for improvement of the team in the accessibility of users to childcare consultation, starting from new studies referring to the main related barriers and actions to be instituted to expand the population's access in the monitoring of the child.
\end{abstract}

Keywords: Primary health care; Child care; Health evaluation. 


\section{Resumen}

El estudio tuvo como objetivo evaluar la calidad del acceso de primer contacto en la consulta puericultura en una Estrategia de Salud de la Familia (ESF) desde la perspectiva de los cuidadores de niños, utilizando PCATool. Estudio descriptivo y transversal, con enfoque cuantitativo desarrollado en la ESF de un municipio de la Fronteira-Oeste del Rio Grande do Sul, Brasil. Participaron 34 cuidadores, responsable de los niños de 0 a 5 años y que realizan cuidado infantil y asisten a la unidad. La mayoría eran madres o abuelos $(97 \%)$, casadas $(70,6 \%)$, un hijo $(32,4 \%)$ y casa propia $(82,4 \%)$. Políticas fomentan los servicios para promover consultas de cuidado infantil que lleguen a todos los niños. La fragilidad en el acceso de la población se puede ver en otros estudios que traen dificultades de movilidad y en programar el día y horario de consulta como principales motivos de baja adherencia. El acceso en cuanto a la atención, tiempo para obtener la consulta y dificultad para obtener la atención médica obtuvo, en la gran mayoría, respuestas satisfactorias. El tiempo de espera y asesoría telefónica obtuvieron resultados inferiores a los esperados, planteando la hipótesis de que son motivos por los que las familias pueden optar por no continuar. Existe la necesidad de mejorar el equipo en la accesibilidad de los usuarios a la consulta de cuidado infantil, con nuevos estudios referentes a las principales barreras relacionadas y acciones para ampliar el acceso de la población en el seguimiento del niño.

Palabras clave: Atención primaria de salud; Cuidado del niño; Evaluación en salud.

\section{Introdução}

O Plano Nacional de Saúde (PNS) trata-se de um instrumento que planeja e conduz a avaliação das políticas e programas do Ministério da Saúde (MS) e organiza diretrizes e metas a serem cumpridas para orientar a coordenação do Sistema Único de Saúde (SUS), permitindo a ampliação e qualidade no uso de seus princípios (Brasil, 2020a).

No âmbito da saúde da criança, o PNS apontou que no período compreendido entre 2016 e 2019 foram registradas 4.933.284 internações pediátricas com suas principais causas sendo evitáveis por meio de cuidados básicos de saúde. Apesar disso, dados do IBGE indicam que a taxa de mortalidade infantil apresenta uma propensão de queda desde a década de 90. Ainda assim, 41,4\% das mortes de crianças entre 1 e 9 anos de idade acontecem devido a acidentes comuns na infância que estão relacionados às condições assistenciais ofertadas na Atenção Primária à Saúde (APS) (Brasil, 2020a, 2020b, 2019).

A APS é definida como uma forma de organizar os serviços de saúde, priorizando a qualidade de vida de cada indivíduo, através da prestação do cuidado integral e do acesso universal. É considerada a entrada preferencial para a assistência de todas as necessidades e problemas que a população possa enfrentar em todos os níveis de atenção (Brasil, 2017).

O acompanhamento sistemático do crescimento e desenvolvimento do neonato, criança e adolescente, oportuniza ao profissional de saúde da APS propor intervenções focadas no cuidado integral, na promoção da saúde e na prevenção de problemas de saúde centrados na família. Surge como uma ferramenta da APS para alcançar objetivos de redução de morbimortalidade, desnutrição, lesões não intencionais, obesidade, dentre outros (Fazeni, et al., 2020).

Para atingir seus eixos estratégicos, a APS adota atributos considerados essenciais para as ações e serviços em saúde, como: acesso do primeiro contato do usuário com o serviço; longitudinalidade; integralidade; e coordenação da atenção (Brasil, 2020c; Starfield, 2002). Como forma de avaliar a qualidade da APS e de seu serviço prestado, o instrumento de Avaliação da Atenção Primária - Primary Care Assessment Tool (PCATool) - Brasil mensura os atributos essenciais (acesso de primeiro contato, longitudinalidade, coordenação e integralidade) e atributos derivados (orientação familiar e orientação comunitária). Neste estudo utilizou-se PCATool - Brasil para pacientes criança na versão extensa, sendo que o instrumento é aplicado junto ao cuidador principal, ou seja, aquele que costuma acompanhar a criança para as consultas de puericultura nos serviços de APS, pois presume-se que este cuidador tem o maior conhecimento do cuidado prestado à saúde da criança (Brasil, 2020c).

Avaliando-se especificamente o acesso de primeiro contato, no que se refere a acessibilidade aos serviços de saúde, dado que em 2015, através da Portaria n 1.130, o MS instituiu a Política Nacional de Atenção Integral à Saúde da Criança (PNAISC), objetivando expandir a promoção e proteção à saúde da criança e o aleitamento materno a partir dos seus eixos estratégicos e diretrizes ${ }^{8}$. A política ainda conta com oito princípios baseados no SUS, sendo o terceiro deles o acesso universal 
à saúde, que pode ser compreendido como a ausência de barreiras socioculturais, demográficas e econômicas para o cuidado à saúde da população (Paim, 2020).

Ainda, a PNAISC visa contemplar o atendimento e acompanhamento da saúde da criança de uma forma integral e de qualidade, assistindo esse público conforme seu crescimento e desenvolvimento, a fim de se tornar um adulto saudável e minimizar as necessidades de internações hospitalares. Para isso, a política estimula os serviços de APS para que promovam as consultas de puericultura de forma que atinja todas as crianças de sua população adscrita (Brasil, 2015, 2018).

A partir disso, este estudo teve por objetivo avaliar a qualidade do acesso de primeiro contato na consulta de puericultura em uma Estratégia Saúde da Família a partir da perspectiva de cuidadores de crianças, utilizando o PCAToolBrasil.

\section{Metodologia}

Trata-se de um estudo descritivo, transversal, de abordagem quantitativa desenvolvido por integrantes bolsistas do Programa de Educação Tutorial Práticas Integradas em Saúde Coletiva (PET PISC) vinculado à Universidade Federal do Pampa. O PET PISC é um Programa de ensino, pesquisa e extensão com atividades voltadas à promoção da saúde coletiva e prevenção de agravos e doenças na comunidade. A partir das ações do PET PISC estabeleceu-se vínculo com uma Unidade de Saúde da Família, na qual existem duas Equipes de Saúde da Família, de um município da fronteira oeste do Rio Grande do Sul, para desenvolvimento das atividades acadêmicas no local, além da implementação da pesquisa.

A unidade de saúde selecionada conta com duas equipes Saúde da Família (ESF), das quais incluem: dois médicos, dois enfermeiros, três técnicos de enfermagem e um Agente Comunitário de Saúde (ACS), além de uma recepcionista e uma auxiliar de higienização. A unidade está localizada em bairro próximo ao Centro da cidade, entretanto, a população adstrita caracteriza-se por famílias em situação de vulnerabilidade social, idosos, hipertensos, diabéticos e usuários com outras comorbidades crônicas, conforme dados cadastrados na ESF. Para atender as demandas de saúde desta população há programas e atividades como o Sistema de Cadastramento e Acompanhamento de Hipertensos e Diabéticos (Hiperdia), consulta de puericultura, acompanhamento do pré-natal de baixo risco, Programa Saúde na Escola (PSE) e Programa Nacional de Imunização (PNI), entre outros. Tais ações são realizadas conforme dinâmica e calendário específico da unidade.

Os atendimentos ocorrem por demanda espontânea e programada, geralmente, a demanda espontânea no turno da manhã, para que os usuários com necessidades de saúde possam ser avaliados em consulta com profissional de saúde. E a demanda programada referente à puericultura, ocorre em dois dias na semana para implementação das consultas com enfermeira e médico. O acompanhamento do crescimento e desenvolvimento ocorre por meio de busca ativa das crianças, sendo a busca realizada através de dados do pré-natal de gestantes cadastradas na unidade, e a partir da demanda espontânea no serviço de saúde.

Em um primeiro momento, foi realizada uma reunião dos pesquisadores com a Enfermeira da equipe 1 para explicar as etapas da pesquisa e dos recursos necessários para realização da mesma. Destes recursos, foi solicitada uma sala reservada, com mesa e cadeiras, para que os pesquisadores pudessem realizar as entrevistas sem interrupções externas e garantindo o anonimato e privacidade dos participantes. Além disso, foi acordado com a profissional que a captação dos participantes ocorreria em duas etapas: busca de endereço dos usuários nos registros do Teste de Triagem Neonatal Biológica (teste do pezinho) realizadas na ESF, sendo realizada visita ao domicílio junto com o ACS; e pela captação de participantes nos dias da consulta de puericultura realizada na unidade.

Os critérios de inclusão utilizados foram: ser familiar ou principal cuidador de crianças entre zero e cinco anos de idade; que possuam maior vínculo com a criança e/ou que costumam levá-la nas consultas de puericultura; cadastrados na Unidade de Saúde da Família selecionada; com realização de pelo menos uma consulta de puericultura desde o nascimento; e 
que concordasse em participar da pesquisa. Foram excluídos da pesquisa os cuidadores de crianças que utilizavam o serviço de saúde de forma esporádica ou que levaram a criança apenas uma vez na unidade, sem continuidade de acompanhamento. Participaram do estudo 34 cuidadores de crianças, com idade entre zero à cinco anos.

A primeira etapa do estudo foi realizada com a busca de alguns endereços na base de dados da unidade de crianças que realizaram o Teste de Triagem Neonatal Biológica. Após, com os endereços determinados, os pesquisadores deslocaram-se até as residências e os cuidadores foram então convidados a participar do presente estudo. Nos casos de divergências nos endereços, os pesquisadores se deslocavam até a ESF selecionada nos dias em que estava planejado no calendário da unidade a realização da consulta de puericultura.

A pesquisa foi aprovada pelo Comitê de Ética em Pesquisa da Universidade local sob o registro CAAE nº 4.176.069. A identidade dos participantes foi preservada, a partir do codinome "A", seguido de numeração aleatória. A coleta dos dados foi realizada de agosto à dezembro de 2018. Após realização da consulta de puericultura com a Enfermeira, a mesma encaminhava os cuidadores que referiam disponibilidade de participar do estudo para outra sala, na qual era apresentado TCLE e explicado cada etapa da pesquisa. Cada entrevista teve tempo de duração de cerca de 20 a 30 minutos. As respostas do instrumento foram registradas em cartão-resposta disponibilizado pelo mesmo.

Os dados foram coletados utilizando o Instrumento de Avaliação da Atenção Primária (PCATool)- Brasil versão para pacientes criança versão estendida, validado no Brasil e disponível pelo Ministério da Saúde. O instrumento avalia ao total 55 questões com base nos atributos essenciais e derivados de uma unidade de saúde, podendo ser de escolha do pesquisador quais utilizar, uma vez que o instrumento é de livre adaptação. Neste estudo utilizou-se seis questões que fazem parte do atributo "Acesso de Primeiro Contato - Acessibilidade", as quais encontram-se na Tabela 1.

Tabela 1 - Perguntas para avaliação do atributo Acesso de Primeiro Contato - Acessibilidade, conforme instrumento Avaliação da Atenção Primária (PCATool- Brasil)

C1 - Quando a ESF está aberta e sua criança fica doente, alguém deste serviço de saúde a atende no mesmo dia?

C2 - Você tem que esperar muito tempo ou falar com muitas pessoas para marcar hora na ESF?

C3- É fácil marcar hora para uma consulta de rotina na ESF?

C4- Quando você chega na ESF, você tem que esperar mais de 30 minutos para que sua criança consulte com o médico/enfermeiro (sem contar triagem ou acolhimento)?

C5- É difícil para você conseguir atendimento médico para sua criança na ESF?

C6- Quando a ESF está aberta, você consegue aconselhamento rápido pelo telefone se precisar?

Fonte: PCATool Brasil/2020 - Manual do instrumento de Avaliação da Atenção Primária à Saúde (2020), 44-45.

Para cada item, as respostas estão dispostas conforme uma Escala de Likert, identificadas como "(4) com certeza sim”, “(3) provavelmente sim”, “(2) provavelmente não”, “(1) com certeza não” e “(9) não sei/não lembro”. Além do PCATool, foi elaborado um pequeno instrumento semiestruturado para coletar dados sociodemográficos dos participantes, contemplando o grau de filiação do cuidador com a criança, estado civil, número de filhos e se possui casa própria.

Para uma avaliação completa da APS, o instrumento orienta que sejam realizadas as entrevistas utilizando-se de todos os componentes que formam os atributos essenciais e derivados. Assim, pode-se calcular o Escore Geral da APS. Entretanto, como no presente estudo foi realizada apenas a aplicação do atributo Acesso de Primeiro Contato - Acessibilidade, os dados foram analisados conforme escore calculado para o atributo proposto no Manual PCATool- Brasil/2020(Brasil, 2020c). 
Seguindo as orientações do manual, para cálculo apenas do atributo selecionado, foram seguidos três passos: o primeiro passo é a reversão dos valores das respostas dos pesquisados, uma vez que em alguns itens, o número máximo (4) não significa que seja o melhor resultado. Portanto, o PCATool versão criança orienta que nos itens C2, C4 e C5 sejam revertidos os valores da seguinte forma $(4=1)(3=2)(2=3)(1=4)$ (Brasil, 2020c).

O segundo passo refere-se àquelas respostas de "Não sei/Não lembro", orientando que sejam anuladas se as mesmas atingirem mais de $50 \%$ das respostas do atributo ou transformadas para valor " 2 " se atingirem menos de $50 \%$. Como último e $3^{\circ}$ passo para cálculo de escore do atributo Acesso de Primeiro Contato - Acessibilidade, é realizada a soma da média dos itens $(\mathrm{C} 1+\mathrm{C} 2+\mathrm{C} 3+\mathrm{C} 4+\mathrm{C} 5+\mathrm{C} 6)$ dividido pelo número de componentes (6). A partir do número obtido, pode-se converter para escala de 0 a 10, conforme fórmula apresentada no manual do PCATool-Brasil - 2020 (Brasil, 2020c)

O resultado obtido pode ser classificado em "Alto" (escore $\geq 6,6$ ) e "Baixo" (escore $<6,6$ ), sendo "Alto" considerado que a unidade básica de saúde selecionada contempla os componentes do atributo avaliado, revelando serviços reconhecidos como orientados à APS (Brasil, 2020c). Após o cálculo do escore, os dados obtidos foram tabulados no programa Microsoft Office Excel e passaram por processo analítico através do software SPSS para avaliação da média, desvio-padrão e valores mínimos e máximos de cada componente do atributo a fim de discussão dos resultados.

Destaca-se que os riscos do estudo foram mínimos e trataram-se de cansaço físico ou constrangimento ao responder algum questionamento, sendo ofertado pausa, água e em caso de constrangimento participante foi esclarecido que suas respostas não prejudicariam seu atendimento na Unidade de saúde e seria garantido seu anonimato. Quanto aos benefícios para os participantes destaca-se que ao avaliar o acesso de primeiro contato ofertado, se pode aprimorar este acesso ofertado pela Unidade de Saúde aos pacientes criança e seus cuidadores.

\section{Resultados}

Foram entrevistados, ao total, 34 cuidadores de crianças entre zero e cinco anos que realizavam puericultura, que estavam cadastrados na ESF selecionada e que utilizavam o serviço com frequência, não apenas esporadicamente. Na Tabela 2 pode-se observar as características sociodemográficas dos pesquisados, sendo que a maioria eram mães (23) e avós (10) totalizando 97\%(33), tendo apenas um (1) pai entrevistado (2,9\%). Além disso, quanto ao estado civil dos cuidadores prevaleceu o casado $(70,6 \%)(24)$. Quanto ao número de filhos 58,9\% (20) dos entrevistados tiveram até dois filhos. No que se refere à moradia, a maioria relatou possuir casa própria $(82,4 \%)(28)$. 
Tabela 2 - Perfil sociodemográfico dos participantes da pesquisa cadastrados em ESF da Fronteira-Oeste do Rio Grande do Sul, Brasil.

\begin{tabular}{|c|c|c|}
\hline Variáveis & $\mathbf{N}$ & $\%$ \\
\hline Cuidador principal & - & - \\
\hline Mãe & 23 & 67,6 \\
\hline Avó & 10 & 29,4 \\
\hline Pai & 1 & 2,9 \\
\hline Estado Civil & - & - \\
\hline Casado & 24 & 70,6 \\
\hline Solteiro & 10 & 29,4 \\
\hline$N^{o}$ de filhos & - & - \\
\hline Um filho & 11 & 32,4 \\
\hline Dois filhos & 9 & 26,5 \\
\hline Três filhos & 4 & 11,8 \\
\hline Quatro filhos & 4 & 11,8 \\
\hline Cinco filhos & 2 & 5,9 \\
\hline Seis filhos & 3 & 8,8 \\
\hline Oito filhos & 1 & 2,9 \\
\hline Casa própria & - & - \\
\hline Sim & 28 & 82,4 \\
\hline Não & 6 & 17,6 \\
\hline
\end{tabular}

Fonte: Dados da pesquisa (2020).

O número de respostas obtidas para cada componente do atributo a partir da pesquisa realizada e suas respectivas porcentagens estão demonstradas na Tabela 3. Quando questionados se os cuidadores recebem atendimento do serviço de saúde no mesmo dia em que sua criança fica doente, 73,5\% (25) responderam que com certeza sim, entretanto, 17,6\%(6) optaram por com certeza não. Em relação ao tempo que os indivíduos levam para conseguir marcar hora na ESF, 55,9\%(19) dos entrevistados afirmaram que com certeza não precisam esperar muito tempo ou falar com muitas pessoas para tal. Ainda assim, 32,4\% (27) dos cuidadores apontaram entre com certeza sim e provavelmente sim.

Ao questionar se é fácil marcar hora para uma consulta de rotina na ESF, 67,6\% (23) dos participantes afirmaram que com certeza sim. Ainda, nesse item, foi possível perceber que a discordância não teve grande impacto, atingindo 11,8\%(4) das respostas “com certeza não". O item referente ao tempo de espera após chegada na ESF (sem contar triagem ou acolhimento) até o atendimento com o profissional de saúde (médico ou enfermeiro) obteve maior equiparidade. Dos entrevistados, 29,4\%(10) afirmaram que com certeza sim aguardam mais de 30 minutos para serem atendidos. Por outro lado, 23,5\% (8) referem que com certeza não.

Acerca da dificuldade de conseguir um atendimento médico para seu filho na ESF, 55,9\% (19) afirmaram que não é difícil marcar consulta para suas crianças. Ainda, 11,8\% (4) responderam que com certeza sim. Por fim, 55,9\%(19) dos cuidadores responderam que com certeza não conseguem aconselhamento rápido pelo telefone se precisar. Somado a isso, 20,6\%(7) dos entrevistados não souberam responder, muitos declarando não saber se há telefone na unidade ou se a equipe costuma realizar este tipo de aconselhamento. 
Tabela 3 - Distribuição de frequência dos itens do atributo Acesso de Primeiro Contato - Acessibilidade na perspectiva de cuidadores de crianças referente à consulta de puericultura.

\begin{tabular}{|c|c|c|c|c|c|c|c|c|c|c|}
\hline \multicolumn{11}{|c|}{ Acesso de Primeiro Contato - Acessibilidade } \\
\hline \multirow[t]{2}{*}{ Itens } & \multirow{2}{*}{$\begin{array}{c}\begin{array}{l}\text { Com } \\
\operatorname{sim}(4)\end{array} \\
n\end{array}$} & \multirow{2}{*}{$\begin{array}{c}\text { certeza } \\
\%\end{array}$} & \multicolumn{2}{|c|}{$\begin{array}{l}\text { Provavelmente } \\
\operatorname{sim}(3)\end{array}$} & \multicolumn{2}{|c|}{$\begin{array}{l}\text { Provavelmente } \\
\text { não (2) }\end{array}$} & \multirow{2}{*}{$\begin{array}{c}\text { Com } \\
\text { não (1) } \\
\text { n }\end{array}$} & certeza & \multicolumn{2}{|c|}{$\begin{array}{l}\text { Não sei/Não } \\
\text { lembro }(9)\end{array}$} \\
\hline & & & $\mathbf{n}$ & $\%$ & $\mathbf{n}$ & $\%$ & & $\%$ & $\mathbf{N}$ & $\%$ \\
\hline C1 & 25 & 73,5 & 2 & 5,9 & 1 & 2,9 & 6 & 17,6 & 0 & 0 \\
\hline $\mathrm{C2}$ & 4 & 11,8 & 7 & 20,6 & 3 & 8,8 & 19 & 55,9 & 1 & 2,9 \\
\hline C3 & 23 & 67,6 & 3 & 8,8 & 3 & 8,8 & 4 & 11,8 & 1 & 2,9 \\
\hline $\mathrm{C4}$ & 10 & 29,4 & 9 & 26,5 & 6 & 17,6 & 8 & 23,5 & 1 & 2,9 \\
\hline C5 & 4 & 11,8 & 4 & 11,8 & 6 & 17,6 & 19 & 55,9 & 1 & 2,9 \\
\hline C6 & 5 & 14,7 & 0 & 0 & 3 & 8,8 & 19 & 55,9 & 7 & 20,6 \\
\hline
\end{tabular}

Fonte: Dados da pesquisa (2020).

A Tabela 4 apresenta a associação das respostas obtidas em cada componente do atributo selecionado com os valores médios por item e total, desvio-padrão por item e total, valor mínimo e valor máximo utilizados. Além disso, conforme orientação do instrumento PCATool, foi realizado o cálculo do escore por item e total, apresentando valor de 6,16, o que se refere à um escore total Baixo $(<6,6)$.

Analisando os escores obtidos para cada componente, constata-se que a maior parte dos itens obtiveram escore Alto $(\geq 6,6)$, o que significa que o acesso de primeiro contato referente à acessibilidade dos cuidadores de crianças na consulta de puericultura da ESF pesquisada apresenta-se, em sua grande maioria, satisfatório conforme o orientado aos serviços de APS. Entretanto, os itens C4 e C6 que se referem, respectivamente, ao tempo de espera superior à 30 minutos desde que a criança chega à unidade de saúde até o seu atendimento com o médico/enfermeiro e ao aconselhamento rápido ao telefone em casos de necessidade e que a ESF esteja aberta, apresentaram-se com escore Baixo $(<6,6)$.

Tabela 4 - Valores médios, desvios-padrão, mínimos e máximos para cada item do atributo Acesso de Primeiro Contato Acessibilidade do instrumento PCATool.

\begin{tabular}{lccccc}
\hline Item & Média por item & Escore por item* & DP** & Mín*** & \multicolumn{2}{c}{ Máx**** } \\
\hline C1 & 3,35 & 7,83 & 1,17 & 1 & 4 \\
\hline C2 & 3,08 & 6,93 & 1,13 & 1 & 4 \\
\hline C3 & 3,36 & 7,86 & 1,08 & 1 & 4 \\
\hline C4 & 2,36 & 4,53 & 1,16 & 1 & 4 \\
\hline C5 & 3,21 & 7,36 & 1,08 & 1 & 4 \\
\hline C6 & 1,73 & 2,43 & 1,05 & 1 & 4 \\
\hline Média total & $\mathbf{2 , 8 5}$ & - & 1,11 & 1 & 4 \\
\hline Escore total & - & $\mathbf{6 , 1 6}$ & - & - & -
\end{tabular}

*Escala de 0 a 10 para definir escore Alto $(\geq 6,6)$ ou Baixo $(<6,6), * *$ Desvio-padrão, ***Valor mínimo, ****Valor máximo. Fonte: Dados da pesquisa (2020). 


\section{Discussão}

Ainda assim, estudo realizado por Vitolo, Gama e Campagnolo (2010) demonstrou que 53,2\% das crianças com idades entre 12 e 16 meses não foram acompanhadas regularmente em consultas de puericultura. Grande parte dos motivos relatados pelos cuidadores das crianças avaliados foram em relação a achar a consulta desnecessária ou por problemas no serviço, levando à falta de acesso dessas famílias às consultas. Rezer, Souza e Faustino (2020) e Gauterio, Irala e Cezar-Vaz (2012) apontam que o dia e horário da consulta, a falta de conhecimento sobre a puericultura e a dificuldade de locomoção (por questões financeiras e de acessibilidade) foram os principais motivos pelos quais cuidadores de crianças interromperam o acompanhamento de seus filhos em unidade básica de saúde. Portanto, o fácil acesso da população aos serviços de saúde, embora seja um dos pilares da PNAISC, vem demonstrando fragilidades, principalmente quando referido ao acompanhamento da saúde da criança na APS.

A implementação de um instrumento validado no Brasil e que proporciona a avaliação de componentes e atributos na APS permitiu verificar a execução e expansão das ações realizadas pelos serviços de saúde em relação à acessibilidade nas consultas de puericultura a partir da visão de usuários cadastrados. Com base nos resultados obtidos, pode-se analisar que o acesso dos indivíduos referente ao atendimento no mesmo dia em que a criança encontra-se doente (desde que a unidade esteja aberta), o tempo aguardado para conseguir marcar hora em consultas rotineiras e não-rotineiras e a dificuldade para conseguir atendimento médico para seus filhos obtiveram, em sua grande maioria, respostas satisfatórias. Isso se deve, principalmente, pela dinâmica organizada na ESF pesquisada, uma vez em que há dias na semana específicos para serem realizadas as consultas por livre demanda e consultas de acompanhamento da criança.

A Política Nacional de Atenção Básica (PNAB) é reconhecida como uma das principais políticas para coordenar a atenção em saúde no âmbito do SUS. A mesma é utilizada por todas unidades básicas de saúde como modelo assistencial e gerencial de suas ações. Com isso, a mesma traz que para o correto funcionamento do processo de trabalho das equipes da unidade deve ser realizado o acolhimento do usuário com escuta ativa, classificação de risco, avaliação das necessidades de saúde e análise da vulnerabilidade do núcleo familiar e não apenas do sujeito. Além disso, deve prover atenção integral e contínua à população adscrita, enfatizando como as ESFs devem organizar-se referente à demanda dos usuários cadastrados (Brasil, 2017).

Por outro lado, a PNAB ainda traz que a prática do cuidado familiar e dirigido à coletividade é necessária para influência do processo saúde-doença dos indivíduos, proporcionando um atendimento humanizado, organizado e responsabilizando-se pela continuidade da atenção para viabilização do vínculo entre profissionais-comunidade. Ainda, se há falhas no seguimento do cuidado, a equipe deve estar apta à realização de buscas dos indivíduos através das visitas domiciliares, incentivando os mesmos a dar prosseguimento nos seus cuidados (Brasil, 2017; Soares, 2016). Com base nisso, quando os itens C4 e C6 avaliados da pesquisa obtiveram baixo escore $(<6,6)$, levanta-se a hipótese de o fato do tempo de espera ser maior que 30 minutos para aguardar uma consulta e não haver aconselhamento rápido por telefone na unidade, serem motivos dos quais as famílias possam optar por não dar continuidade às consultas de puericultura.

Ademais, muitos cuidadores de crianças acabam deixando de levá-las ao acompanhamento no serviço básico de saúde por não achar que há necessidade, uma vez que a criança se encontra sadia, tal comportamento poderia ser relacionado ao modelo de saúde baseado na doença e não no acompanhamento de ciclos de vida e na promoção da saúde. Entretanto, a puericultura está inserida na agenda de compromissos do MS para a promoção da saúde integral da criança e redução da mortalidade infantil, visando acompanhar o crescimento e desenvolvimento da criança através de uma avaliação completa e na detecção precoce de possíveis alterações, e não apenas quando a criança se encontra doente (Zanardo et al., 2017).

Destaca-se como limitação deste estudo a avaliação apenas do acesso de primeiro contato no que diz respeito a acessibilidade aos serviços de saúde, sendo necessário avaliar outros atributos da APS relacionada à saúde da criança para 
promover um olhar ampliado à saúde da criança.

\section{Considerações Finais}

Através do estudo, verificou-se a necessidade de aprimoramento da equipe referente ao acesso dos usuários à consulta de puericultura, uma vez que, barreiras como falta de acesso à informação, horários e dias de funcionalidade inflexíveis, como também desconhecimento dos cuidadores sobre a importância do acompanhamento da saúde da criança.

Sugere-se que a partir dos resultados obtidos no presente estudo, a pesquisa possa servir de fomento para a realização de nova análise da qual busque os principais motivos dos quais cuidadores de crianças relatam estar relacionados à baixa acessibilidade das consultas de puericultura na ESF estudada. Além disso, propõe-se ações instituídas às equipes de saúde da unidade direcionadas para qualificação do cuidado à puericultura, incluindo o acesso, organização e gestão do serviço, avaliação das consultas, engajamento da equipe e ampliação das atividades que promovam o conhecimento da população frente à relevância do acompanhamento do crescimento e desenvolvimento da criança na Atenção Básica.

Destaca-se a necessidade de ampliação do estudo para avaliação de outros atributos, tais como a longitudinalidade do cuidado à saúde da criança e outras dimensões do instrumento que poderiam caracterizar pontos fortes e fragilidades no perfil de atenção à criança.

\section{Referências}

Fanezi, L. N., Getelina, C. O., Rotoli, A., \& Alves, R. da R. (2020). Características de crianças atendidas em consulta de puericultura. Research, Society and Development, 9(4), e154943022. https://doi.org/10.33448/rsd-v9i4.3022

Gauterio, D. P., Irala, D. de A., \& Cezar-Vaz, Marta Regina. (2012). Puericultura em Enfermagem: perfil e principais problemas encontrados em crianças menores de um ano. Revista Brasileira de Enfermagem, 65(3), 508-513. https://doi.org/10.1590/S0034-71672012000300017

Manual do instrumento de avaliação da atenção primária à saúde: Primary Care Assessment Tool: PCATool-Brasil: Secretaria de Atenção à Saúde, Departamento de Atenção Básica.

Ministério da Economia. Tábua completa de mortalidade para o Brasil - 2018: breve análise da evolução da mortalidade no Brasil: IBGE, Coordenação de População e Indicadores Sociais.

Ministério da Saúde. Plano Nacional de Saúde 2020-2023. Agência Nacional de Vigilância Sanitária - ANVISA: Ministério da Saúde.

Ministério da Saúde. Portaria n ${ }^{\circ} 1.130$ de 05 de agosto de 2015. Institui a Política Nacional de Atenção Integral à Saúde da Criança (PNAISC) no âmbito do Sistema Único de Saúde (SUS). Brasília, DF. https://bvsms.saude.gov.br/bvs/ saudelegis/gm /2015/prt1130_05_08_2015.html

Ministério da Saúde. Portaria no 2.436, de 21 de setembro de 2017. Política Nacional de Atenção Básica: Diário Oficial da União.

Ministério da Saúde. Sistema de Informações sobre Mortalidade. Banco de dados do Sistema Único de Saúde - DATASUS. Informações de Saúde.

Paim, J. S. (2020). Os sistemas universais de saúde e o futuro do Sistema Único de Saúde (SUS). Saúde em Debate [online], 43(spe5), 15-28. doi.org/10.1590/0103-11042019S50

Política Nacional de Atenção Integral à Saúde da Criança: orientações para implementação: Ministério da Saúde.

Rezer, F., Souza, T. V., \& Faustino, W. R. (2020). Dificuldades dos responsáveis por crianças na adesão à puericultura. Journal Health NPEPS, 5(1), 338-350.

Soares, L. T. (2016). Adesão às consultas de puericultura na área de abrangência da ESF Turmalina I em Governador Valadares. Trabalho de Conclusão de Curso, Especialização em Saúde da Família, Universidade Federal de Minas Gerais. Polo Belo Horizonte, Brasil.

Starfield, B. (2002). Starfield, Barbara Atenção primária: equill̀brio entre necessidades de saúde, serviços e tecnologia. Brasìlia : UNESCO, Ministério da Saúde, 2002. 726p. https://www.nescon.medicina.ufmg.br/biblioteca/imagem/0253.pdf

Vitolo, M. R., Gama, C. M., \& Campagnolo, P. D. B. (2010). Frequency of public child care service use and associated factors. J Pediatr, 86(1), 80-84.

Zanardo, G., Andrade, U., Zanardo, G., \& Menezes, L. P. (2017). Atuação do enfermeiro na consulta de puericultura: uma revisão narrativa de literatura. Revista de Enfermagem, 13(13). 